\title{
Superconvergence of Semidiscrete Splitting Positive Definite Mixed Finite Elements for Hyperbolic Optimal Control Problems
}

\author{
Yuchun Hua and Yuelong Tang \\ Institute of Computational Mathematics, College of Science, Hunan University of Science and Engineering, Yongzhou, \\ 425100 Hunan, China \\ Correspondence should be addressed to Yuelong Tang; tangyuelonga@163.com
}

Received 5 September 2021; Accepted 17 December 2021; Published 6 January 2022

Academic Editor: Leopoldo Greco

Copyright (c) 2022 Yuchun Hua and Yuelong Tang. This is an open access article distributed under the Creative Commons Attribution License, which permits unrestricted use, distribution, and reproduction in any medium, provided the original work is properly cited.

In this paper, we consider semidiscrete splitting positive definite mixed finite element methods for optimal control problems governed by hyperbolic equations with integral constraints. The state and costate are approximated by the lowest order Raviart-Thomas mixed rectangular finite element, and the control is approximated by piecewise constant functions. We derive some convergence and superconvergence results for the control, the state and the adjoint state. A numerical example is provided to demonstrate our theoretical results.

\section{Introduction}

There have been extensive studies in error estimates of standard finite element methods (FEMs) for optimal control problems (OCPs). The convergence or superconvergence results of standard FEMs for elliptic and parabolic OCPs can be found in [1-13], respectively.

Because large temperature gradients during cooling or heating may lead to its destruction in temperature control problems, the gradient stands for Darcy velocity in flow control problems, stiffness optimization in nonlinear pantographic structures [14], and topology optimization of a cycloidal metamaterial [15]; their objective functionals contain not only the primal state variable but also its gradient. At this time, mixed finite element methods (MFEMs) will be a very good choice for solving this kind of OCPs. The convergence or superconvergence results of MFEMs for elliptic and parabolic OCPs can be found in [16-20], respectively. However, mixed finite element spaces have to satisfy the Ladyženskaja-Babuška-Brezzi (LBB) condition, which brings very little available approximation spaces and expensive computing costs.

In order to avoid the limitation of LBB condition, a splitting positive definite MFEM was first proposed for solving miscible displacement of compressible flow in a porous medium [21]. Compared with the classic MFEMs, the main advantages of this method are that the original problems can be split into two independent symmetric positive definite subschemes and that the LBB condition is not necessary. Recently, splitting positive definite MFEMs have been used to solve hyperbolic equations [22, 23], elliptic OCPs [24], and parabolic OCPs [25]. To our best knowledge, most of the published papers on different FEMs or MFEMs for OCPs are focused on elliptic or parabolic cases. Although $\mathrm{Xu}$ in [26] established a priori error estimates and superconvergence results of splitting positive definite MFEM for pseudohyperbolic integrodifferential OCPs and Lu et al. in [27] derived the convergence of finite volume element method for nonlinear hyperbolic OCPs, there are very little studies on hyperbolic OCPs, .

The goal of this paper is to investigate splitting positive definite MFEMs for hyperbolic OCPs and derive the convergence and superconvergence.

We are interested in the following hyperbolic OCPs:

$$
\min _{u \in K}\left\{\frac{1}{2} \int_{0}^{T}\left(\left\|\boldsymbol{p}-\boldsymbol{p}_{d}\right\|^{2}+\left\|y-y_{d}\right\|^{2}+\|u\|^{2}\right) d t\right\},
$$




$$
\begin{gathered}
c(x) y_{t t}(x, t)+\operatorname{div} \boldsymbol{p}(x, t)=f(x, t)+u(x, t), x \in \Omega, t \in J, \\
\boldsymbol{p}(x, t)=-A(x) \nabla y(x, t), x \in \Omega, t \in J \\
y(x, t)=0, x \in \partial \Omega, t \in J \\
y(x, 0)=y_{0}(x), y_{t}(x, 0)=y_{1}(x), x \in \Omega
\end{gathered}
$$

where $\Omega \subset \mathbf{R}^{2}$ is a rectangle domain, $J=(0, T], \quad \boldsymbol{p}_{d} \in$ $\left(L^{2}\left(J ; L^{2}(\Omega)\right)\right)^{2}, y_{d}, f \in L^{2}\left(J ; L^{2}(\Omega)\right), c \in W^{1, \infty}(\Omega)$ with 0 $<c_{\min } \leq c(x) \leq c_{\max }$ and $y_{0}, y_{1} \in H^{2}(\Omega)$. The coefficient matrix $A(x)=\left(a_{i j}(x)\right)_{2 \times 2} \in W^{1, \infty}\left(\bar{\Omega} ; \mathbf{R}^{2 \times 2}\right)$ is a symmetric matrix, and there are constants $c_{1}, c_{2}>0$ satisfying any vector $\mathbf{X} \in \mathbf{R}^{2}, c_{1}\|\mathbf{X}\|_{\mathbf{R}^{2}}^{2} \leq \mathbf{X}^{t} A \mathbf{X} \leq c_{2}\|\mathbf{X}\|_{\mathbf{R}^{2}}^{2}$. $K$ is a set defined by

$$
K=\left\{u \in L^{2}\left(J ; L^{2}(\Omega)\right): \int_{0}^{T} \int_{\Omega} u d x d t \geq 0\right\}
$$

In this paper, we adopt the standard notation $W^{m, p}(\Omega)$ for Sobolev spaces on $\Omega$ with a norm $\|\cdot\|_{m, p}$ given by $\|v\|_{m, p}^{p}=\sum_{|\alpha| \leq m}\left\|D^{\alpha} v\right\|_{L^{p}(\Omega)}^{p}$, a seminorm $|\cdot|_{m, p}$ given by $|v|_{m, p}^{p}$ $=\sum_{|\alpha|=m}\left\|D^{\alpha} v\right\|_{L^{p}(\Omega)}^{p}$. For $p=2$, we set $H^{m}(\Omega)=W^{m, 2}(\Omega)$, $H_{0}^{1}(\Omega)=\left\{v \in H^{1}(\Omega):\left.v\right|_{\partial \Omega}=0\right\}$, and $\|\cdot\|_{m}=\|\cdot\|_{m, 2},\|\cdot\|=$ $\|\cdot\|_{0,2}$. We denote by $L^{s}\left(J ; W^{m, p}(\Omega)\right)$ the Banach space of all $L^{s}$ integrable functions from $J$ into $W^{m, p}(\Omega)$ with norm $\|v\|_{L^{s}\left(J ; W^{m, p}(\Omega)\right)}=\left(\int_{0}^{T}\|v\|_{W^{m, p}(\Omega)}^{s} d t\right)^{1 / s}$ for $s \in[1, \infty)$, and the standard modification for $s=\infty$. For simplicity of presentation, we denote $\|v\|_{L^{s}\left(J ; W^{m, p}(\Omega)\right)}$ by $\|v\|_{L^{s}\left(W^{m, p}\right)}$. Similarly, one can define the spaces $H^{1}\left(J ; W^{m, p}(\Omega)\right)$ and $C^{k}\left(J ; W^{m, p}(\Omega)\right)$ . In addition, $C$ denotes a general positive constant independent of $h$, where $h$ is the spatial mesh-size for the control and state discretization.

The plan of this paper is as follows. In Section 2, we give an equivalent optimality conditions for the OCP (1)-(5) and construct its splitting positive definite mixed finite element approximation scheme. In Section 3, we derive the convergence for the control variable, the state variables, and the adjoint state variables. In Section 4, we derive the superconvergence properties between the $R T$ projections and the approximation solutions of the control and the state variables. In the last section, we present a numerical example to illustrate our theoretical results.

\section{Splitting Positive Definite MFEMs for OCPs}

In this section, we shall construct a splitting positive definite mixed finite element approximation of the control problems (1)-(5). To fix the idea, we shall take the state spaces $\mathbf{L}=$ $H^{2}(J ; \boldsymbol{V})$ and $Q=H^{2}(J ; W)$, where $\boldsymbol{V}$ and $W$ are defined as follows.

Let

$$
\boldsymbol{V}=H(\operatorname{div} ; \Omega)=\left\{\boldsymbol{v} \in\left(L^{2}(\Omega)\right)^{2}, \operatorname{div} \boldsymbol{v} \in L^{2}(\Omega)\right\}, W=L^{2}(\Omega),
$$

and the inner products

$$
\begin{gathered}
\left(f_{1}, f_{2}\right)=\int_{\Omega} f_{1} f_{2}, \forall f_{1}, f_{2} \in L^{2}(\Omega), \\
(\varphi, \psi)=\sum_{i=1}^{2}\left(\varphi_{i}, \psi_{i}\right), \forall \varphi, \psi \in\left(L^{2}(\Omega)\right)^{2} .
\end{gathered}
$$

Let $b=1 / c(x)$, a mixed weak form of (2), and (3) can be given by

$$
\left(A^{-1} \boldsymbol{p}, \boldsymbol{v}\right)=(y, \operatorname{div} \boldsymbol{v}), \forall \boldsymbol{v} \in \boldsymbol{V}, t \in J
$$

$$
\left(y_{t t}, w\right)+(b \operatorname{div} \boldsymbol{p}, w)=(b f, w)+(b u, w), \forall w \in W, t \in J
$$

As in [24], taking $w=\operatorname{div} \mathbf{v}, \forall \mathbf{v} \in \mathbf{V}$ in (10), (9) differentiating twice with respect to $t$, and then substituting the two resulting equations, we derive

$$
\left(A^{-1} \boldsymbol{p}_{t t}, \boldsymbol{v}\right)+(b \operatorname{div} \boldsymbol{p}, \operatorname{div} \boldsymbol{v})=(b f, \operatorname{div} \boldsymbol{v})+(b u, \operatorname{div} \boldsymbol{v}), \forall \boldsymbol{v} \in \boldsymbol{V}, t \in J .
$$

By using (10) and (11), we get the following new mixed variational form:

$$
\left(A^{-1} \boldsymbol{p}_{t t}, \boldsymbol{v}\right)+(b \operatorname{div} \boldsymbol{p}, \operatorname{div} \boldsymbol{v})=(b f, \operatorname{div} \boldsymbol{v})+(b u, \operatorname{div} \boldsymbol{v}), \forall \mathbf{v} \in \mathbf{V}, t \in J,
$$

$\left(y_{t t}, w\right)+(b \operatorname{div} \boldsymbol{p}, w)=(b f, w)+(b u, w), \forall w \in W, t \in J$

It is easily seen that (12) is separated from (13) so that $p$ can be solved independently from (12).

We recast (1)-(5) as the following weak form: find ( $\boldsymbol{p}$, $y, u) \in \mathbf{L} \times Q \times K$ such that

$$
\begin{gathered}
\min _{u \in K}\left\{\frac{1}{2} \int_{0}^{T}\left(\left\|\boldsymbol{p}-\boldsymbol{p}_{d}\right\|^{2}+\left\|y-y_{d}\right\|^{2}+\|u\|^{2}\right) d t\right\} \\
\left(A^{-1} \boldsymbol{p}_{t t}, \boldsymbol{v}\right)+(b \operatorname{div} \boldsymbol{p}, \operatorname{div} \boldsymbol{v})=(b f, \operatorname{div} \boldsymbol{v})+(b u, \operatorname{div} \boldsymbol{v}), \forall \boldsymbol{v} \in \boldsymbol{V}, t \in J \\
\boldsymbol{p}(x, 0)=-A \nabla y_{0}(x), \boldsymbol{p}_{t}(x, 0)=-A \nabla y_{1}(x), \forall x \in \Omega \\
\left(y_{t t}, w\right)+(b \operatorname{div} \boldsymbol{p}, w)=(b f, w)+(b u, w), \forall w \in W, t \in J \\
y(x, 0)=y_{0}(x), y_{t}(x, 0)=y_{1}(x), \forall x \in \Omega
\end{gathered}
$$

It then follows from [28] that the optimal control problems (14)-(18) have a unique solution $(p, y, u)$, and that a triplet $(p, y, u)$ is the solution of (14)-(18) if and only if there is a costate $(\boldsymbol{q}, z) \in \mathbf{L} \times Q$ such that $(\boldsymbol{p}, y, \boldsymbol{q}, z, u)$ satisfies the 
following optimality conditions:

$\left(A^{-1} \boldsymbol{p}_{t t}, \boldsymbol{v}\right)+(b \operatorname{div} \boldsymbol{p}, \operatorname{div} \boldsymbol{v})=(b f, \operatorname{div} \boldsymbol{v})+(b u, \operatorname{div} \boldsymbol{v}), \forall \boldsymbol{v} \in \boldsymbol{V}, t \in J$

$$
\boldsymbol{p}(x, 0)=-A \nabla y_{0}(x), \boldsymbol{p}_{t}(x, 0)=-A \nabla y_{1}(x), \forall x \in \Omega,
$$

$\left(y_{t t}, w\right)+(b \operatorname{div} \boldsymbol{p}, w)=(b f, w)+(b u, w), \forall w \in W, t \in J$,

$$
y(x, 0)=y_{0}(x), y_{t}(x, 0)=y_{1}(x), \forall x \in \Omega,
$$

$\left(A^{-1} \boldsymbol{q}_{t t}, \boldsymbol{v}\right)+(b \operatorname{div} \boldsymbol{p}, \operatorname{div} \boldsymbol{v})+(b z, \operatorname{div} \boldsymbol{v})=-\left(\boldsymbol{p}-\boldsymbol{p}_{d}, \boldsymbol{v}\right), \forall \boldsymbol{v} \in \boldsymbol{V}, t \in J$

$$
\begin{gathered}
\boldsymbol{q}(x, T)=0, \boldsymbol{q}_{t}(x, T)=0, \forall x \in \Omega, \\
\left(z_{t t}, w\right)=-\left(y-y_{d}, w\right), \forall w \in W, t \in J, \\
z(x, T)=0, z_{t}(x, T)=0, \forall x \in \Omega, \\
\int_{0}^{T}(u-b z-b \operatorname{div} \boldsymbol{q}, \tilde{u}-u) d t \geq 0, \forall \tilde{u} \in K .
\end{gathered}
$$

The inequality (27) can be expressed as

$$
u=\max \{0,-\bar{G}\}+G,
$$

where $G=(b z+b \operatorname{div} \boldsymbol{q})$ and $\bar{G}=\int_{0}^{T} \int_{\Omega} G d x d t /(|\Omega| \times T)$.

Let $\boldsymbol{T}_{h}$ be a uniform rectangulation of the domain $\Omega$, and $h_{e}$ denotes the diameter of element $e$ and $h=\max _{e \in T_{h}}\left\{h_{e}\right\}$. Let $\boldsymbol{V}_{h} \times W_{h} \subset \boldsymbol{V} \times W$ denote the lowest order Raviart-Thomas mixed finite element space $[29,30]$, namely

$$
\begin{gathered}
\boldsymbol{V}_{h}:=\left\{\boldsymbol{v}_{h} \in \boldsymbol{V}: \forall e \in \boldsymbol{T}_{h},\left.\boldsymbol{v}_{h}\right|_{e} \in Q_{1,0}(e) \times Q_{0,1}(e)\right\}, \\
W_{h}:=\left\{w_{h} \in W: \forall e \in \boldsymbol{T}_{h},\left.w_{h}\right|_{e} \in Q_{0,0}(e)\right\},
\end{gathered}
$$

where $Q_{m, n}(e)$ indicates the space of polynomials of degree no more than $m$ and $n$ in $x_{1}$ and $x_{2}$ on $e$, respectively. And the approximated space of control is given by

$$
K_{h}:=L^{2}\left(J ; W_{h}\right) \cap K
$$

We introduce two projection operators. First, we define the standard $L^{2}(\Omega)$-projection [29] $P_{h}: W \longrightarrow W_{h}$, which satisfies the following: for any $\phi \in W$

$$
\begin{gathered}
\left(P_{h} \phi-\phi, w_{h}\right)=0, \forall w_{h} \in W_{h} \\
\left\|\phi-P_{h} \phi\right\|_{-s, \rho} \leq C h^{1+s}\|\phi\|_{1, \rho}, s=0,1,2 \leq \rho \leq \infty, \forall \phi \in W^{1, \rho}(\Omega) .
\end{gathered}
$$

Second, we recall the Fortin projection (see $[29,31]$ ) $\Pi_{h}: \boldsymbol{V} \longrightarrow \boldsymbol{V}_{h}$, which satisfies the following: for any $\boldsymbol{q} \in \boldsymbol{V}$

$$
\left(\operatorname{div}\left(\Pi_{h} \boldsymbol{q}-\boldsymbol{q}\right), w_{h}\right)=0, \forall w_{h} \in W_{h},
$$

$$
\left\|\mathbf{q}-\Pi_{h} \mathbf{q}\right\|_{0, \rho} \leq C h\|\mathbf{q}\|_{1, \rho}, 2 \leq \rho \leq \infty, \forall \boldsymbol{q} \in\left(W^{1, \rho}(\Omega)\right)^{2},
$$

$\left\|\operatorname{div}\left(\boldsymbol{q}-\Pi_{h} \boldsymbol{q}\right)\right\| \leq C h\|\operatorname{div} \boldsymbol{q}\|_{1}, \forall \operatorname{div} \boldsymbol{q} \in H^{1}(\Omega)$.

Then the splitting positive definite mixed finite element discretization of (14)-(18) is as follows: find $\left(\boldsymbol{p}_{h}, y_{h}, u_{h}\right) \in$ $H^{2}\left(J ; V_{h}\right) \times H^{2}\left(J ; W_{h}\right) \times K_{h}$ such that

$$
\begin{gathered}
\min _{u_{h} \in K_{h}}\left\{\frac{1}{2} \int_{0}^{T}\left(\left\|\boldsymbol{p}_{h}-\boldsymbol{p}_{d}\right\|^{2}+\left\|y_{h}-y_{d}\right\|^{2}+\left\|u_{h}\right\|^{2}\right) d t\right\} \\
\left(A^{-1} \boldsymbol{p}_{h, t t}, \boldsymbol{v}_{h}\right)+\left(b \operatorname{div} \boldsymbol{p}_{h}, \operatorname{div} \boldsymbol{v}_{h}\right)=\left(b f, \operatorname{div} \boldsymbol{v}_{h}\right) \\
+\left(b u_{h}, \operatorname{div} \boldsymbol{v}_{h}\right), \forall \boldsymbol{v}_{h} \in \boldsymbol{V}_{h}, t \in J
\end{gathered}
$$

The optimal control problems (36)-(40) again have a unique solution $\left(\boldsymbol{p}_{h}, y_{h}, u_{h}\right)$, and a triplet $\left(\boldsymbol{p}_{h}, y_{h}, u_{h}\right)$ is the solution of (36)-(40) if and only if there is a costate $\left(\mathbf{q}_{h}, z_{h}\right)$ such that $\left(\boldsymbol{p}_{h}, y_{h}, \boldsymbol{q}_{h}, z_{h}, u_{h}\right)$ satisfies the following optimality conditions:

$$
\begin{gathered}
\left(A^{-1} \boldsymbol{p}_{h, t t}, \boldsymbol{v}_{h}\right)+\left(b \operatorname{div} \boldsymbol{p}_{h}, \operatorname{div} \boldsymbol{v}_{h}\right)=\left(b f, \operatorname{div} \boldsymbol{v}_{h}\right) \\
+\left(b u_{h}, \operatorname{div} \boldsymbol{v}_{h}\right), \forall \boldsymbol{v}_{h} \in \boldsymbol{V}_{h}, t \in J \\
\boldsymbol{p}_{h}(x, 0)=\Pi_{h} \boldsymbol{p}(x, 0), \boldsymbol{p}_{h, t}(x, 0)=\Pi_{h} \boldsymbol{p}_{t}(x, 0), \forall x \in \Omega, \\
\left(y_{h, t t}, w_{h}\right)+\left(b \operatorname{div} \boldsymbol{p}_{h}, w_{h}\right)=\left(b f, w_{h}\right)+\left(b u_{h}, w_{h}\right), \forall w_{h} \in W_{h}, t \in J, \\
y_{h}(x, 0)=P_{h} y_{0}(x), y_{h, t}(x, 0)=P_{h} y_{0}(x), \forall x \in \Omega, \\
\left(A^{-1} \boldsymbol{q}_{h, t t}, \boldsymbol{v}_{h}\right)+\left(b \operatorname{div} \boldsymbol{q}_{h}, \operatorname{div} \boldsymbol{v}_{h}\right)+\left(b z_{h}, \operatorname{div} \boldsymbol{v}_{h}\right) \\
=-\left(\boldsymbol{p}_{h}-\boldsymbol{p}_{d}, \boldsymbol{v}_{h}\right), \forall \boldsymbol{v}_{h} \in \boldsymbol{V}_{h}, t \in J, \\
\boldsymbol{q}_{h}(x, T)=0, \boldsymbol{q}_{h, t}(x, T)=0, \forall x \in \Omega, \\
\left(z_{h, t t}, w_{h}\right)=-\left(y_{h}-y_{d}, w_{h}\right), \forall w_{h} \in W_{h}, t \in J \\
z_{h}(x, T)=0, z_{h, t}(x, T)=0, \forall x \in \Omega,
\end{gathered}
$$

$\int_{0}^{T}\left(u_{h}-b z_{h}-b \operatorname{div} \mathbf{q}_{h}, \tilde{u}_{h}-u_{h}\right) d t \geq 0, \forall \tilde{u}_{h} \in K_{h}$.

Similar to (28), we have

$$
u_{h}=\max \left\{0,-\bar{G}_{h}\right\}+G_{h}
$$

where $G_{h}=P_{h} b\left(z_{h}+\operatorname{div} \boldsymbol{q}_{h}\right)$ and $\bar{G}_{h}=\int_{0}^{T} \int_{\Omega} G_{h} d x d t /(|\Omega| \times$ T). 


\section{Convergence Analysis}

In this section, we will derive the convergence of splitting positive definite MFEMs for hyperbolic OCPs. For $\forall \tilde{u} \in K$, we define the discrete state solution $\left(\boldsymbol{p}_{h}(\tilde{u}), y_{h}(\tilde{u}), \boldsymbol{q}_{h}(\tilde{u}), z_{h}\right.$ $(\tilde{u}))$ associated with $\tilde{u}$ which satisfies

$$
\begin{gathered}
\left(A^{-1} \boldsymbol{p}_{h, t t}(\tilde{u}), \boldsymbol{v}_{h}\right)+\left(b \operatorname{div} \boldsymbol{p}_{h}(\tilde{u}), \operatorname{div} \boldsymbol{v}_{h}\right) \\
=\left(b f, \operatorname{div} \boldsymbol{v}_{h}\right)+\left(b \tilde{u}, \operatorname{div} \boldsymbol{v}_{h}\right), \forall \boldsymbol{v}_{h} \in \boldsymbol{V}_{h}, t \in J, \\
\boldsymbol{p}_{h}(\tilde{u})(x, 0)=\Pi_{h} \boldsymbol{p}(x, 0), \boldsymbol{p}_{h, t}(\tilde{u})(x, 0)=\Pi_{h} \boldsymbol{p}_{t}(x, 0), \forall x \in \Omega,
\end{gathered}
$$

$\left(y_{h, t t}(\tilde{u}), w_{h}\right)+\left(b \operatorname{div} \boldsymbol{p}_{h}(\tilde{u}), w_{h}\right)=\left(b f, w_{h}\right)+\left(b \tilde{u}, w_{h}\right), \forall w_{h} \in W_{h}, t \in J$,

$$
y_{h}(\tilde{u})(x, 0)=P_{h} y_{0}(x), y_{h, t}(\tilde{u})(x, 0)=P_{h} y_{1}(x), \forall x \in \Omega,
$$

$\left(A^{-1} \boldsymbol{q}_{h, t t}(\tilde{u}), \boldsymbol{v}_{h}\right)+\left(b \operatorname{div} \boldsymbol{q}_{h}(\tilde{u}), \operatorname{div} \boldsymbol{v}_{h}\right)+\left(b z_{h}(\tilde{u}), \operatorname{div} \boldsymbol{v}_{h}\right)$

$=-\left(\mathrm{p}_{h}(\tilde{u})-\boldsymbol{p}_{d}, \boldsymbol{v}_{h}\right), \forall \boldsymbol{v}_{h} \in \boldsymbol{V}_{h}, t \in J$,

$$
\begin{gathered}
\boldsymbol{q}_{h}(\tilde{u})(x, T)=0, \boldsymbol{q}_{h, t}(\tilde{u})(x, T)=0, \forall x \in \Omega, \\
\left(z_{h, t t}(\tilde{u}), w_{h}\right)=-\left(y_{h}(\tilde{u})-y_{d}, w_{h}\right), \forall w_{h} \in W_{h}, t \in J, \\
z_{h}(\tilde{u})(x, T)=0, z_{h, t}(\tilde{u})(x, T)=0, \forall x \in \Omega .
\end{gathered}
$$

It is clear that the exact solution and its approximation can be written in the following way:

$$
\begin{gathered}
(\boldsymbol{p}, y, \boldsymbol{q}, z)=(\boldsymbol{p}(u), y(u), \boldsymbol{q}(u), z(u)), \\
\left(\boldsymbol{p}_{h}, y_{h}, \boldsymbol{q}_{h}, z_{h}\right)=\left(\boldsymbol{p}_{h}\left(u_{h}\right), y_{h}\left(u_{h}\right), \boldsymbol{q}_{h}\left(u_{h}\right), z_{h}\left(u_{h}\right)\right) .
\end{gathered}
$$

Lemma 1. Let $(\boldsymbol{p}, y, \boldsymbol{q}, z)$ be the solution of (19)-(26) and $\left(\boldsymbol{p}_{h}(u), y_{h}(u), \boldsymbol{q}_{h}(u), z_{h}(u)\right)$ be the solution of (51)-(58) with $\tilde{u}=u$, respectively. If the solution satisfies

$$
\boldsymbol{p}, \boldsymbol{q} \in\left[L^{\infty}\left(H^{1}\right)\right]^{2} \cap\left[L^{2}\left(H^{2}\right)\right]^{2} \cap\left[H^{2}\left(H^{1}\right)\right]^{2} \text { and } y, z \in L^{\infty}\left(H^{1}\right),
$$

then we have

$$
\begin{gathered}
\left\|y-y_{h}(u)\right\|_{L^{\infty}\left(L^{2}\right)}+\left\|\boldsymbol{p}-\boldsymbol{p}_{h}(u)\right\|_{L^{\infty}\left(L^{2}\right)} \leq C h, \\
\left\|z-z_{h}(u)\right\|_{L^{\infty}\left(L^{2}\right)}+\left\|\boldsymbol{q}-\boldsymbol{q}_{h}(u)\right\|_{L^{\infty}\left(L^{2}\right)} \leq C h, \\
\left\|\operatorname{div}\left(\boldsymbol{p}-\boldsymbol{p}_{h}(u)\right)\right\|_{L^{2}\left(L^{2}\right)}+\left\|\operatorname{div}\left(\boldsymbol{q}-\boldsymbol{q}_{h}(u)\right)\right\|_{L^{2}\left(L^{2}\right)} \leq C h .
\end{gathered}
$$

Proof. For ease of presentation, we set $\rho_{\mathbf{p}}=\Pi_{h} \boldsymbol{p}-\boldsymbol{p}_{h}(u)$, $\rho_{y}=P_{h} y-y_{h}(u), \rho_{\boldsymbol{q}}=\Pi_{h} \boldsymbol{q}-\boldsymbol{q}_{h}(u), \rho_{z}=P_{h} z-z_{h}(u)$. From Equations (19)-(26) and (51)-(58) and the fact that div $V_{h} \subset W_{h}$, with the definition of $P_{h}$ and $\Pi_{h}$, we can easily obtain the following error equations:

$$
\begin{aligned}
\left(A^{-1} \rho_{\boldsymbol{p}, t t}, \boldsymbol{v}_{h}\right) & +\left(b \operatorname{div} \rho_{\boldsymbol{p}}, \operatorname{div} \boldsymbol{v}_{h}\right)=-\left(b \operatorname{div}\left(\boldsymbol{p}-\Pi_{h} \boldsymbol{p}\right), \operatorname{div} \boldsymbol{v}_{h}\right) \\
& -\left(A^{-1}\left(\boldsymbol{p}_{t t}-\Pi_{h} \boldsymbol{p}_{t t}\right), \boldsymbol{v}_{h}\right), \forall \boldsymbol{v}_{h} \in \boldsymbol{V}_{h},
\end{aligned}
$$

$$
\begin{aligned}
\left(\rho_{y, t t}, w_{h}\right) & +\left(b \operatorname{div} \rho_{\boldsymbol{p}}, w_{h}\right)=-\left(b \operatorname{div}\left(\boldsymbol{p}-\Pi_{h} \boldsymbol{p}\right), w_{h}\right) \\
& -\left(y_{t t}-P_{h} y_{t t}, w_{h}\right), \forall w_{h} \in W_{h},
\end{aligned}
$$

$$
\begin{aligned}
\left(A^{-1} \rho_{\boldsymbol{q}, t t}, \boldsymbol{v}_{h}\right) & +\left(b \operatorname{div} \rho_{\boldsymbol{p}}, \operatorname{div} \boldsymbol{v}_{h}\right)+\left(b \operatorname{div}\left(\boldsymbol{q}-\Pi_{h} \boldsymbol{q}\right), \operatorname{div} \boldsymbol{v}_{h}\right) \\
& +\left(b\left(z-P_{h} z\right), \operatorname{div} \boldsymbol{v}_{h}\right)=-\left(A^{-1}\left(\boldsymbol{q}_{t t}-\Pi_{h} \boldsymbol{q}_{t t}\right), \boldsymbol{v}_{h}\right) \\
& -\left(\boldsymbol{p}-\Pi_{h} \boldsymbol{p}, \boldsymbol{v}_{h}\right)-\left(\rho_{\boldsymbol{p}}, \boldsymbol{v}_{h}\right)-\left(b \rho_{z}, \operatorname{div} \boldsymbol{v}_{h}\right), \forall \boldsymbol{v}_{h} \in \boldsymbol{V}_{h},
\end{aligned}
$$

$$
\left(\rho_{z, t t}, w_{h}\right)=-\left(\rho_{y}, w_{h}\right)-\left(z_{t t}-P_{h} z_{t t}, w_{h}\right), \forall w_{h} \in W_{h}
$$

Setting $\boldsymbol{v}_{h}=\rho_{p, t}$ in (62), we have

$$
\begin{aligned}
\frac{1}{2} \frac{d}{d t}\left(\left\|A^{-\frac{1}{2}} \rho_{p, t}\right\|^{2}+\left\|b^{\frac{1}{2}} \operatorname{div} \rho_{p}\right\|^{2}\right)= & -\left(b \operatorname{div}\left(\boldsymbol{p}-\Pi_{h} \boldsymbol{p}\right), \operatorname{div} \rho_{p, t}\right) \\
& -\left(A^{-1}\left(\boldsymbol{p}_{t t}-\Pi_{h} \boldsymbol{p}_{t t}\right), \rho_{\boldsymbol{p}, t}\right) .
\end{aligned}
$$

Notice that

$\left(b \operatorname{div}\left(\boldsymbol{p}-\Pi_{h} \boldsymbol{p}\right), \operatorname{div} \rho_{\boldsymbol{p}, t}\right)=-\left(b \operatorname{div}\left(\boldsymbol{p}-\Pi_{h} \boldsymbol{p}\right)_{t}, \operatorname{div} \rho_{\boldsymbol{p}}\right)$.

Substitute (67) into (66), integrating the resulting equation from 0 to $t$, and using Hölder's inequality, Young's inequality, Gronwall's inequality, and the assumption on $A$ and (34) and (35), we have

$$
\left\|\rho_{p, t}\right\|_{L^{\infty}\left(L^{2}\right)}+\left\|\operatorname{div} \rho_{\boldsymbol{p}}\right\|_{L^{\infty}\left(L^{2}\right)} \leq C h .
$$

Letting $w_{h}=\rho_{y, t}$ in (63) and $w_{h}=-\rho_{z, t}$ in (65), respectively. Then integrating the resulting equations from 0 to $t$ and $t$ to $T$, respectively, we get

$$
\begin{gathered}
\left\|\rho_{y, t}\right\|_{L^{\infty}\left(L^{2}\right)} \leq C\left\|\operatorname{div} \rho_{p}\right\|_{L^{2}\left(L^{2}\right)}+C h, \\
\left\|\rho_{z, t}\right\|_{L^{\infty}\left(L^{2}\right)} \leq C\left\|\rho_{y}\right\|_{L^{2}\left(L^{2}\right)}+C h,
\end{gathered}
$$

where we also used Hölder's inequality, Young's inequality, and Gronwall's inequality.

At last, setting $\mathbf{v}_{h}=-\rho_{\mathbf{q}, t}$ as the test function in (64) and integrating the resulting equation from $t$ to $T$, similar to 
(68), we arrive at

$$
\left\|\rho_{\mathbf{q}, t}\right\|_{L^{\infty}\left(L^{2}\right)}+\left\|\operatorname{div} \rho_{\boldsymbol{p}}\right\|_{L^{\infty}\left(L^{2}\right)} \leq C h+C\left(\left\|\rho_{\boldsymbol{p}}\right\|_{L^{2}\left(L^{2}\right)}+\left\|\rho_{z}\right\|_{L^{2}\left(L^{2}\right)}\right) .
$$

Note that $\rho_{\mathbf{p}}(0)=\rho_{y}(0)=\rho_{\boldsymbol{q}}(T)=\rho_{z}(T)=0$, and we have

$$
\begin{aligned}
& \left\|\rho_{\boldsymbol{p}}\right\|_{L^{\infty}\left(L^{2}\right)} \leq C\left\|\rho_{\mathbf{p}, t}\right\|_{L^{2}\left(L^{2}\right)}, \\
& \left\|\rho_{y}\right\|_{L^{\infty}\left(L^{2}\right)} \leq C\left\|\rho_{y, t}\right\|_{L^{2}\left(L^{2}\right)}, \\
& \left\|\rho_{\mathbf{q}}\right\|_{L^{\infty}\left(L^{2}\right)} \leq C\left\|\rho_{\boldsymbol{q}, t}\right\|_{L^{2}\left(L^{2}\right)}, \\
& \left\|\rho_{z}\right\|_{L^{\infty}\left(L^{2}\right)} \leq C\left\|\rho_{z, t}\right\|_{L^{2}\left(L^{2}\right)},
\end{aligned}
$$

Combining (68)-(74), (32), (34), and (35) and the triangle inequality, we complete the proof.

Using the same estimates as in Lemma 1, we get the following.

Lemma 2. Let $\left(\boldsymbol{p}_{h}, y_{h}, \boldsymbol{q}_{h}, z_{h}\right)$ and $\left(\boldsymbol{p}_{h}(u), y_{h}(u), \boldsymbol{q}_{h}(u), z_{h}(u)\right)$ be the solutions of (51)-(58) with $\tilde{u}=u_{h}$ and $\tilde{u}=u$, respectively. Then we have

$$
\begin{gathered}
\left\|y_{h}-y_{h}(u)\right\|_{L^{\infty}\left(L^{2}\right)}+\left\|\boldsymbol{p}_{h}-\boldsymbol{p}_{h}(u)\right\|_{L^{\infty}\left(L^{2}\right)} \leq C\left\|u-u_{h}\right\|_{L^{2}\left(L^{2}\right)}, \\
\left\|z_{h}-z_{h}(u)\right\|_{L^{\infty}\left(L^{2}\right)}+\left\|\boldsymbol{q}_{h}-\boldsymbol{q}_{h}(u)\right\|_{L^{\infty}\left(L^{2}\right)} \leq C\left\|u-u_{h}\right\|_{L^{2}\left(L^{2}\right)}, \\
\left\|\operatorname{div}\left(\boldsymbol{p}_{h}-\boldsymbol{p}_{h}(u)\right)\right\|_{L^{2}\left(L^{2}\right)}+\left\|\operatorname{div}\left(\boldsymbol{q}_{h}-\boldsymbol{q}_{h}(u)\right)\right\|_{L^{2}\left(L^{2}\right)} \leq C\left\|u-u_{h}\right\|_{L^{2}\left(L^{2}\right)} .
\end{gathered}
$$

Now, from the above Lemmas 1 and 2, we can derive the following convergence results.

Theorem 3. Let $u$ be the solution of (19)-(27) and $u_{h}$ be the solution of (41)-(49), respectively. Assume that all the assumptions in Lemma 1 are valid. Then we have

$$
\begin{gathered}
\left\|u-u_{h}\right\|_{L^{2}\left(L^{2}\right)} \leq C h, \\
\left\|y-y_{h}\right\|_{L^{\infty}\left(L^{2}\right)}+\left\|\boldsymbol{p}-\boldsymbol{p}_{h}\right\|_{L^{\infty}\left(L^{2}\right)} \leq C h, \\
\left\|z-z_{h}\right\|_{L^{\infty}\left(L^{2}\right)}+\left\|\boldsymbol{q}-\boldsymbol{q}_{h}\right\|_{L^{\infty}\left(L^{2}\right)} \leq C h,
\end{gathered}
$$

$\left\|\operatorname{div}\left(\boldsymbol{p}-\boldsymbol{p}_{h}\right)\right\|_{L^{2}\left(L^{2}\right)}+\left\|\operatorname{div}\left(\boldsymbol{q}-\boldsymbol{q}_{h}\right)\right\|_{L^{2}\left(L^{2}\right)} \leq C h$.

Proof. Let $w_{h}=1$ in (31), and we have

$$
\int_{\Omega} P_{h} u d x=\int_{\Omega} u d x
$$

By (80), we have

$$
\int_{0}^{T} \int_{\Omega} P_{h} u d x d t=\int_{0}^{T} \int_{\Omega} u d x d t \geq 0
$$

Thus, we know that $P_{h} u \in K_{h}$.

It follows from (27) and (49) that

$$
\begin{aligned}
\left\|u-u_{h}\right\|_{L^{2}\left(L^{2}\right)}^{2}= & \int_{0}^{T}\left(u-u_{h}, u-u_{h}\right) d t=\int_{0}^{T}\left(u-b z-b \operatorname{div} \boldsymbol{q}, u-u_{h}\right) \\
& \cdot d t+\int_{0}^{T}\left(b z-b z_{h}(u)+b \operatorname{div}\left(\boldsymbol{q}-\boldsymbol{q}_{h}(u)\right), u-u_{h}\right) \\
& \cdot d t+\int_{0}^{T}\left(b z_{h}(u)-b z_{h}+b \operatorname{div}\left(\boldsymbol{q}_{h}(u)-\boldsymbol{q}_{h}\right), u-u_{h}\right) \\
& \cdot d t+\int_{0}^{T}\left(b z_{h}+b \operatorname{div} \boldsymbol{q}_{h}-u_{h}, u-u_{h}\right) \\
& \cdot d t \leq \int_{0}^{T}\left(b z-b z_{h}(u)+b \operatorname{div}\left(\boldsymbol{q}-\boldsymbol{q}_{h}(u)\right), u-u_{h}\right) \\
& \cdot d t-\int_{0}^{T}\left(u-b z-b \operatorname{div} \boldsymbol{q}, u-P_{h} u\right) \\
& \cdot d t+\int_{0}^{T}\left(b z_{h}(u)-b z_{h}+b \operatorname{div}\left(\boldsymbol{q}_{h}(u)-\boldsymbol{q}_{h}\right), u-u_{h}\right) \\
& \cdot d t+\int_{0}^{T}\left(u-u_{h}, u-P_{h} u\right) d t+\int_{0}^{T}\left(b z_{h}-b z_{h}(u)\right. \\
& \left.+b \operatorname{div}\left(\boldsymbol{q}_{h}-\boldsymbol{q}_{h}(u)\right), u-P_{h} u\right) \\
& \cdot d t+\int_{0}^{T}\left(b z_{h}(u)-b z+b \operatorname{div}\left(\boldsymbol{q}_{h}(u)-\boldsymbol{q}\right), u-P_{h} u\right) \\
& \cdot d t:=\sum_{i=1}^{6} I_{i} .
\end{aligned}
$$

Next, we estimate (82) term by term. For $I_{1}$, using Hölder's inequality, Young's inequality, and Lemma 1, we have

$$
I_{1} \leq C h^{2}+\frac{1}{4}\left\|u-u_{h}\right\|_{L^{2}\left(L^{2}\right)}^{2}
$$

From (28), we find that

$$
u-b z-b \operatorname{div} \boldsymbol{q}=\text { const }
$$

Thus

$$
I_{2}=- \text { const } \int_{0}^{T} \int_{\Omega}\left(u-P_{h} u\right) d x d t=0 .
$$

Set $\eta_{\boldsymbol{p}}=\boldsymbol{p}_{h}(u)-\boldsymbol{p}_{h}, \eta_{y}=y_{h}(u)-y_{h}, \eta_{\boldsymbol{q}}=\boldsymbol{q}_{h}(u)-\boldsymbol{q}_{h}$, and $\eta_{z}=z_{h}(u)-z_{h}$; then from (51)-(58), we have the following error equations:

$$
\left(A^{-1} \eta_{p, t t}, \boldsymbol{v}_{h}\right)+\left(b \operatorname{div} \eta_{p}, \operatorname{div} \boldsymbol{v}_{h}\right)=\left(b u-b u_{h}, \operatorname{div} \boldsymbol{v}_{h}\right), \forall \boldsymbol{v}_{h} \in \boldsymbol{V}_{h}, t \in J
$$

$$
\left(\eta_{y, t t}, w_{h}\right)+\left(b \operatorname{div} \eta_{\mathrm{p}}, w_{h}\right)=\left(b u-b u_{h}, w_{h}\right), \forall w_{h} \in W_{h}, t \in J,
$$




$$
\begin{aligned}
\left(A^{-1} \eta_{\boldsymbol{q}, t t}, \boldsymbol{v}_{h}\right) & +\left(b \operatorname{div} \eta_{\boldsymbol{q}}, \operatorname{div} \boldsymbol{v}_{h}\right)=-\left(\eta_{\boldsymbol{p}}, \boldsymbol{v}_{h}\right) \\
& -\left(b \eta_{z}, \operatorname{div} \boldsymbol{v}_{h}\right), \forall \boldsymbol{v}_{h} \in \boldsymbol{V}_{h}, t \in J, \\
\left(\eta_{z, t t}, w_{h}\right) & =-\left(\eta_{y}, w_{h}\right), \forall w_{h} \in W_{h}, t \in J .
\end{aligned}
$$

choosing $\mathbf{v}_{h}=\eta_{\mathbf{q}}$ in (86), $w_{h}=\eta_{z}$ in (87), $\mathbf{v}_{h}=\eta_{\mathbf{p}}$ in (88), and $w_{h}=\eta_{y}$ in (89), respectively. Since $\eta_{\mathbf{p}}(0)=\eta_{y}(0)=\eta_{\mathbf{p}, t}(0)=$ $\eta_{y, t}(0)=\eta_{\mathbf{q}}(T)=\eta_{z}(T)=\eta_{\mathbf{q}, t}(T)=\eta_{z, t}(T)=0, \quad$ integrating the resulting equations from 0 to $T$, we can see that

$$
I_{3}=\int_{0}^{T}\left(\eta_{z}+\operatorname{div} \eta_{q}, b u-b u_{h}\right) d t=-\left\|\eta_{\mathbf{p}}\right\|^{2}-\left\|\eta_{y}\right\|^{2} \leq 0
$$

For $I_{4}$ and $I_{5}$, using Hölder's inequality, Young's inequality, Lemma 2, and (32), we get

$$
\begin{gathered}
I_{4} \leq C h^{2}+\frac{1}{4}\left\|u-u_{h}\right\|_{L^{2}\left(L^{2}\right)}^{2}, \\
I_{5} \leq\left(\left\|\eta_{z}\right\|_{L^{2}\left(L^{2}\right)}+\left\|\operatorname{div} \eta_{q}\right\|_{L^{2}\left(L^{2}\right)}\right)\left\|u-P_{h} u\right\|_{L^{2}\left(L^{2}\right)} \\
\leq\left\|u-u_{h}\right\|_{L^{2}\left(L^{2}\right)}\left\|u-P_{h} u\right\|_{L^{2}\left(L^{2}\right)} \leq C h^{2}+\frac{1}{4}\left\|u-u_{h}\right\|_{L^{2}\left(L^{2}\right)}^{2} .
\end{gathered}
$$

For $I_{6}$, by Hölder's inequality, the triangle inequality, Lemma 1, and (32), we arrive at

$I_{6} \leq\|b\|_{0, \infty}\left(\left\|z_{h}(u)-z\right\|_{L^{2}\left(L^{2}\right)}+\left\|\operatorname{div} \eta_{q}\right\|_{L^{2}\left(L^{2}\right)}\right)\left\|u-P_{h} u\right\|_{L^{2}\left(L^{2}\right)} \leq C h^{2}$.

Combining (82), (83), (85), and (90)-(92), we derive (76). Using (76), Lemmas 1 and 2, and the triangle inequality, we complete the proof.

\section{Superconvergence Properties}

In this section, we will derive some superconvergence properties for the optimal control problems. In order to derive the main results, we need the following lemmas.

Lemma 4. Let $(\boldsymbol{p}, y, \boldsymbol{q}, z)$ be the solution of (19)-(27) and $\left(\boldsymbol{p}_{h}(u), y_{h}(u), \boldsymbol{q}_{h}(u), z_{h}(u)\right)$ be the solution of (51)-(58) with $\tilde{u}=u$, respectively. If the solution satisfies

$$
\boldsymbol{p}, \boldsymbol{q} \in\left[L^{\infty}\left(H^{1}\right)\right]^{2} \cap\left[L^{2}\left(H^{2}\right)\right]^{2} \cap\left[H^{1}\left(H^{2}\right)\right]^{2} \text { and } y, z \in L^{\infty}\left(H^{1}\right),
$$

then we have

$$
\begin{aligned}
& \left\|P_{h} y-y_{h}(u)\right\|_{L^{\infty}\left(L^{2}\right)}+\left\|\Pi_{h} \boldsymbol{p}-\boldsymbol{p}_{h}(u)\right\|_{L^{\infty}\left(L^{2}\right)} \leq C h^{\frac{3}{2}}, \\
& \left\|P_{h} z-z_{h}(u)\right\|_{L^{\infty}\left(L^{2}\right)}+\left\|\Pi_{h} \boldsymbol{q}-\boldsymbol{q}_{h}(u)\right\|_{L^{\infty}\left(L^{2}\right)} \leq C h^{\frac{3}{2}},
\end{aligned}
$$

$$
\left\|\operatorname{div}\left(\Pi_{h} \boldsymbol{p}-\boldsymbol{p}_{h}(u)\right)\right\|_{L^{2}\left(L^{2}\right)}+\left\|\operatorname{div}\left(\Pi_{h} \boldsymbol{q}-\boldsymbol{q}_{h}(u)\right)\right\|_{L^{2}\left(L^{2}\right)} \leq C h^{\frac{3}{2}} .
$$

Proof. At first, for any $\boldsymbol{p} \in \boldsymbol{V}$ and $\boldsymbol{v} \in \boldsymbol{V}_{h}$, by applying the proof of Theorems 4.1, 5.1, and Example 6.2 in [32], we can prove

$$
\left(A^{-1}\left(\boldsymbol{p}-\Pi_{h} \boldsymbol{p}\right), \boldsymbol{v}_{h}\right) \leq C h^{\frac{3}{2}}\|\mathbf{p}\|_{2}\left(\left\|\mathbf{v}_{h}\right\|+\left\|\operatorname{div} \boldsymbol{v}_{h}\right\|\right) .
$$

Moreover, using (32) and (35), we have

$\left(b \operatorname{div}\left(\boldsymbol{p}-\Pi_{h} \boldsymbol{p}\right), \operatorname{div} \boldsymbol{v}_{h}\right)=\left(\left(b-P_{h} b\right) \operatorname{div}\left(\boldsymbol{p}-\Pi_{h} \boldsymbol{p}\right), \operatorname{div} \boldsymbol{v}_{h}\right)$

$$
\leq C h^{2}\|b\|_{1, \infty}\|\mathbf{p}\|_{2}\left\|\operatorname{div} \boldsymbol{v}_{h}\right\|
$$

$$
\begin{aligned}
\left(b\left(y-P_{h} y\right), \operatorname{div} \boldsymbol{v}_{h}\right) & =\left(\left(b-P_{h} b\right)\left(y-P_{h} y\right), \operatorname{div} \boldsymbol{v}_{h}\right) \\
& \leq C h^{2}\|b\|_{1, \infty}\|y\|_{1}\left\|\operatorname{div} \boldsymbol{v}_{h}\right\| .
\end{aligned}
$$

Similar to Lemma 1, using (98), we can prove (95)-(97). We omit the proof here.

Lemma 5. Let $\left(\boldsymbol{p}_{h}\left(P_{h} u\right), y_{h}\left(P_{h} u\right), \boldsymbol{q}_{h}\left(P_{h} u\right), z_{h}\left(P_{h} u\right)\right)$ and $\left(\boldsymbol{p}_{h}\right.$ $\left.(u), y_{h}(u), \boldsymbol{q}_{h}(u), z_{h}(u)\right)$ be the solutions of (51)-(58) with $\tilde{u}=P_{h} u$ and $\tilde{u}=u$, respectively. Then we have

$$
\begin{aligned}
& \left\|y_{h}(u)-y_{h}\left(P_{h} u\right)\right\|_{L^{\infty}\left(L^{2}\right)}+\left\|\boldsymbol{p}_{h}(u)-\boldsymbol{p}_{h}\left(P_{h} u\right)\right\|_{L^{\infty}\left(L^{2}\right)}=0, \\
& \left\|z_{h}(u)-z_{h}\left(P_{h} u\right)\right\|_{L^{\infty}\left(L^{2}\right)}+\left\|\boldsymbol{q}_{h}(u)-\boldsymbol{q}_{h}\left(P_{h} u\right)\right\|_{L^{\infty}\left(L^{2}\right)}=0,
\end{aligned}
$$

$\left\|\operatorname{div}\left(\boldsymbol{p}_{h}(u)-\boldsymbol{p}_{h}\left(P_{h} u\right)\right)\right\|_{L^{2}\left(L^{2}\right)}+\left\|\operatorname{div}\left(\boldsymbol{q}_{h}(u)-\boldsymbol{q}_{h}\left(P_{h} u\right)\right)\right\|_{L^{2}\left(L^{2}\right)}=0$.

Proof. First, set $\zeta_{\boldsymbol{p}}=\boldsymbol{p}_{h}(u)-\boldsymbol{p}_{h}\left(P_{h} u\right), \zeta_{y}=y_{h}(u)-y_{h}\left(P_{h} u\right)$, $\zeta_{\boldsymbol{q}}=\boldsymbol{q}_{h}(u)-\boldsymbol{q}_{h}\left(P_{h} u\right)$, and $\zeta_{z}=z_{h}(u)-z_{h}\left(P_{h} u\right)$, and we choose $\tilde{u}=P_{h} u$ and $\tilde{u}=u$ in (51)-(58), respectively; then we obtain the following error equations:

$\left(A^{-1} \zeta_{p, t}, v_{h}\right)+\left(b \operatorname{div} \zeta_{p}, \operatorname{div} \boldsymbol{v}_{h}\right)=\left(b u-b P_{h} u, \operatorname{div} \boldsymbol{v}_{h}\right), \forall v_{h} \in V_{h}, t \in J$,

$\left(\zeta_{y, t t}, w_{h}\right)+\left(b \operatorname{div} \zeta_{p}, w_{h}\right)=\left(b u-b P_{h} u, w_{h}\right), \forall w_{h} \in W_{h}, t \in J$,

$$
\begin{aligned}
\left(A^{-1}\left(\zeta_{\boldsymbol{q}, t t}, \boldsymbol{v}_{h}\right)\right. & +\left(b \operatorname{div} \zeta_{\boldsymbol{q}}, \operatorname{div} \boldsymbol{v}_{h}\right)=-\left(\zeta_{\boldsymbol{p}}, \boldsymbol{v}_{h}\right) \\
& -\left(b \zeta_{z}, \operatorname{div} \boldsymbol{v}_{h}\right), \forall \boldsymbol{v}_{h} \in \boldsymbol{V}_{h}, t \in J \\
\left(\zeta_{z, t t}, w_{h}\right)= & -\left(\zeta_{y}, w_{h}\right), \forall w_{h} \in W_{h}, t \in J
\end{aligned}
$$

Noting from the fact that $\operatorname{div} \mathbf{V}_{h} \subset W_{h}$ and (31)

$$
\begin{gathered}
\left(b u-b P_{h} u, \operatorname{div} v_{h}\right) \leq C h^{2}\|b\|_{1, \infty}\|u\|_{1}\left\|\operatorname{div} \boldsymbol{v}_{h}\right\|, \forall v_{h} \in V_{h} \\
\left(b u-b P_{h} u, w_{h}\right) \leq C h^{2} h^{2}\|b\|_{1, \infty}\|u\|_{1}\left\|w_{h}\right\|, \forall w_{h} \in W_{h}
\end{gathered}
$$


then, as in Lemma 1, using the stability estimates, we complete the proof.

Lemma 6. Let $\left(\boldsymbol{p}_{h}\left(P_{h} u\right), y_{h}\left(P_{h} u\right), \boldsymbol{q}_{h}\left(P_{h} u\right), z_{h}\left(P_{h} u\right)\right)$ and ( $\left.\boldsymbol{p}_{h}, y_{h}, \boldsymbol{q}_{h}, z_{h}\right)$ be the solutions of (51)-(58) with $\tilde{u}=P_{h} u$ and $\tilde{u}=u_{h}$, respectively. Then we have

$$
\int_{0}^{T}\left[\left(b z_{h}\left(P_{h} u\right)-b z_{h}, P_{h} u-u_{h}\right)+\left(b \operatorname{div}\left(\boldsymbol{q}_{h}\left(P_{h} u\right)-\boldsymbol{q}_{h}\right), P_{h} u-u_{h}\right)\right] d t \leq 0 .
$$

Proof. Set $\theta_{\boldsymbol{p}}=\boldsymbol{p}_{h}-\boldsymbol{p}_{h}\left(P_{h} u\right), \theta_{y}=y_{h}-y_{h}\left(P_{h} u\right), \theta_{\boldsymbol{q}}=\boldsymbol{q}_{h}-\boldsymbol{q}_{h}($ $\left.P_{h} u\right)$, and $\theta_{z}=z_{h}-z_{h}\left(P_{h} u\right)$, similar to (101)-(104), and we obtain the following error equations:

$$
\begin{array}{r}
\left(A^{-1} \theta_{p, t t}, \boldsymbol{v}_{h}\right)+\left(b \operatorname{div}\left(\theta_{\boldsymbol{p}}, \operatorname{div} \boldsymbol{v}_{h}\right)=\left(b u_{h}-b P_{h} u, \operatorname{div} \boldsymbol{v}_{h}\right), \forall \boldsymbol{v}_{h} \in \boldsymbol{V}_{h},\right. \\
\left(\theta_{y, t t}, w_{h}\right)+\left(b \operatorname{div} \theta_{\boldsymbol{p}}, w_{h}\right)=\left(b u_{h}-b P_{h} u, w_{h}\right), \forall w_{h} \in W_{h}, \\
\left(A^{-1}\left(\theta_{\boldsymbol{q}, t t}, \boldsymbol{v}_{h}\right)+\left(b \operatorname{div} \theta_{\boldsymbol{q}}, \operatorname{div} \boldsymbol{v}_{h}\right)=-\left(\theta_{\boldsymbol{p}}, \boldsymbol{v}_{h}\right)+\left(b \theta_{z}, \operatorname{div} \boldsymbol{v}_{h}\right), \forall \boldsymbol{v}_{h} \in \boldsymbol{V}_{h},\right. \\
\left(\theta_{z, t t}, w_{h}\right)=-\left(\theta_{y}, w_{h}\right), \forall w_{h} \in W_{h},
\end{array}
$$

choosing $\boldsymbol{v}_{h}=\theta_{\boldsymbol{q}}$ in (107), $w_{h}=\theta_{z}$ in (108), $\boldsymbol{v}_{h}=-\theta_{\boldsymbol{p}}$ in (109), and $w_{h}=-\theta_{y}$ in (110), respectively. Integrating the resulting equations from 0 to $T$, we derive

$$
\begin{aligned}
& \int_{0}^{T}\left[\left(z_{h}\left(P_{h} u\right)-z_{h}, b P_{h} u-b u_{h}\right)\right. \\
& \left.\quad+\left(\operatorname{div}\left(\boldsymbol{q}_{h}\left(P_{h} u\right)-\boldsymbol{q}_{h}\right), b P_{h} u-b u_{h}\right)\right] \\
& \cdot d t=-\left\|\theta_{y}\right\|_{L^{2}\left(L^{2}\right)}^{2}-\left\|\theta_{p}\right\|_{L^{2}\left(L^{2}\right)}^{2}
\end{aligned}
$$

which yields to (106).

Theorem 7. Let $u$ be the solution of (19)-(27) and $u_{h}$ be the solution of (41)-(49), respectively. Assume that all the assumptions in Lemma 1 are valid. Then, we have

$$
\left\|P_{h} u-u_{h}\right\|_{L^{2}\left(L^{2}\right)} \leq C h^{\frac{3}{2}}
$$

Proof. We choose $\tilde{u}=u_{h}$ in (23) and $\tilde{u}_{h}=P_{h} u$ in (44) to get the following two inequalities:

$$
\begin{gathered}
\int_{0}^{T}\left(u-b z-b \operatorname{div} \boldsymbol{q}, u_{h}-u\right) d t \geq 0, \\
\int_{0}^{T}\left(u_{h}-b z_{h}-b \operatorname{div} \boldsymbol{q}_{h}, P_{h} u-u_{h}\right) d t \geq 0 .
\end{gathered}
$$

Note that $u_{h}-u=u_{h}-P_{h} u+P_{h} u-u$. Adding the above two inequalities, we get

$$
\begin{gathered}
\int_{0}^{T}\left(u_{h}-u+b z-b z_{h}+b \operatorname{div}\left(\boldsymbol{q}-\boldsymbol{q}_{h}\right), P_{h} u-u_{h}\right) \\
\cdot d t+\int_{0}^{T}\left(u-b z-b \operatorname{div} \boldsymbol{q}, P_{h} u-u\right) d t \geq 0 .
\end{gathered}
$$

Thus, by (114) and (26), we find that

$$
\begin{aligned}
&\left\|P_{h} u-u_{h}\right\|_{L^{2}\left(L^{2}\right)=}^{2} \int_{0}^{T}\left(P_{h} u-u, P_{h} u-u_{h}\right) \\
& \cdot \cdot \mathrm{d} t+\int_{0}^{T}\left(u-u_{h}, P_{h} u-u_{h}\right) \\
& \cdot d t \leq \int_{0}^{T}\left(b z-b z_{h}+b \operatorname{div} \boldsymbol{q}-b \operatorname{div} \boldsymbol{q}_{h}, P_{h} u-u_{h}\right) \\
& \cdot d t+\int_{0}^{T}\left(u-b z-b \operatorname{div} \boldsymbol{q}, P_{h} u-u\right) \\
& \cdot d t=\int_{0}^{T}\left(b z-b P_{h} z+b \operatorname{div} \boldsymbol{q}-b \operatorname{div} \Pi_{h} \mathbf{q}, P_{h} u-u_{h}\right) \\
& \cdot d t+\int_{0}^{T}\left(b P_{h} z-b z_{h}(\mathbf{u})+b \operatorname{div} \Pi_{h} \boldsymbol{q}\right. \\
&\left.-\operatorname{div} \boldsymbol{q}_{h}(u), P_{h} u-u_{h}\right) d t+\int_{0}^{T}\left(b z_{h}(u)-b z_{h}\left(P_{h} u\right)\right. \\
&\left.+b \operatorname{div} \boldsymbol{q}_{h}(u)-b \operatorname{div} \boldsymbol{q}_{h}\left(P_{h} u\right), P_{h} u-u_{h}\right) \\
& \cdot d t+\int_{0}^{T}\left(b z_{h}\left(P_{h} u\right)-b z_{h}+b \operatorname{div} \boldsymbol{q}\left(P_{h} u\right)\right. \\
&\left.-b \operatorname{div} \boldsymbol{q}_{h}, P_{h} u-u_{h}\right) d t+\int_{0}^{T}(u-b z \\
&\left.-b \operatorname{div} \boldsymbol{q}, P_{h} u-u\right) d t:=\sum_{i=1}^{5} J_{i} .
\end{aligned}
$$

At first, from Cauchy inequality, (26), (28), and (24), it is easy to get

$$
\begin{aligned}
J_{1}= & \int_{0}^{T}\left(b z-b P_{h} z+b \operatorname{div} \boldsymbol{q}-b \operatorname{div} \Pi_{h} \boldsymbol{q}, P_{h} u-u_{h}\right) \\
& \cdot d t \leq C h^{4}+\frac{1}{4}\left\|P_{h} u-u_{h}\right\|_{L^{2}\left(L^{2}\right)}^{2} .
\end{aligned}
$$

Using Hölder's inequality, Young's inequality, and Lemmas 4 and 5 , we arrive at

$$
\begin{gathered}
J_{2} \leq C h^{3}+\frac{1}{4}\left\|P_{h} u-u_{h}\right\|_{L^{2}\left(L^{2}\right)}^{2}, \\
J_{3} \leq C h^{3}+\frac{1}{4}\left\|P_{h} u-u_{h}\right\|_{L^{2}\left(L^{2}\right)}^{2} .
\end{gathered}
$$

Combining (84), (85), and (115)-(118) and Lemma 6, we derive (112).

Using Theorem 7 and the stability estimates as in Lemma 1, we can arrive at the following. 
TABLE 1: Numerical results of convergence.

\begin{tabular}{ccccccc}
\hline$h=\tau$ & $\left\|e_{u}\right\|_{2,2}$ & Rate & $\left\|e_{y}\right\|_{\infty, 2}$ & Rate & $\left\|e_{z}\right\|_{\infty, 2}$ & Rate \\
\hline $1 / 10$ & $4.9561 e-2$ & - & $3.5081 e-2$ & - & $4.0448 e-2$ & - \\
$1 / 20$ & $2.5280 e-2$ & 0.97 & $1.8105 e-2$ & 0.95 & $2.0416 e-2$ & 0.99 \\
$1 / 40$ & $1.2642 e-2$ & 1.00 & $9.1468 e-3$ & 0.99 & $1.0208 e-2$ & 1.00 \\
$1 / 80$ & $6.2663 e-3$ & 1.01 & $4.5615 e-3$ & 1.00 & $5.1032 e-3$ & 1.00 \\
\hline
\end{tabular}

Lemma 8. Let $\left(\boldsymbol{p}_{h}\left(P_{h} u\right), y_{h}\left(P_{h} u\right), \boldsymbol{q}_{h}\left(P_{h} u\right), z_{h}\left(P_{h} u\right)\right)$ and ( $\left.\boldsymbol{p}_{h}, y_{h}, \boldsymbol{q}_{h}, z_{h}\right)$ be the solutions of (51)-(58) with $\tilde{u}=P_{h} u$ and $\tilde{u}=u_{h}$, respectively. Assume that all the assumptions in Theorem 7 are valid. Then we have

$$
\begin{aligned}
& \left\|y_{h}-y_{h}\left(P_{h} u\right)\right\|_{L^{\infty}\left(L^{2}\right)}+\left\|\boldsymbol{p}_{h}-\boldsymbol{p}_{h}\left(P_{h} u\right)\right\|_{L^{\infty}\left(L^{2}\right)} \leq C h^{\frac{3}{2}}, \\
& \left\|z_{h}-z_{h}\left(P_{h} u\right)\right\|_{L^{\infty}\left(L^{2}\right)}+\left\|\boldsymbol{q}_{\boldsymbol{h}}-\boldsymbol{q}_{\boldsymbol{h}}\left(P_{h} u\right)\right\|_{L^{\infty}\left(L^{2}\right)} \leq C h^{\frac{3}{2}}, \\
& \left\|\operatorname{div}\left(\boldsymbol{p}_{h}-\boldsymbol{p}_{h}\left(P_{h} u\right)\right)\right\|_{L^{2}\left(L^{2}\right)}+\left\|\operatorname{div}\left(\boldsymbol{q}_{h}-\boldsymbol{q}_{h}\left(P_{h} u\right)\right)\right\|_{L^{2}\left(L^{2}\right)} \leq C h^{\frac{3}{2}} .
\end{aligned}
$$

Combining Lemma 4-8 and using the triangle inequality, we derive the following superconvergence results.

Theorem 9. Let $(y, \boldsymbol{p}, z, \boldsymbol{q})$ and $\left(y_{h}, \boldsymbol{p}_{h}, z_{h}, \boldsymbol{q}_{h}\right)$ be the solutions of (19)-(27) and (41)-(49), respectively. Assume that all the assumptions in Theorem 7 are valid. Then we have

$$
\begin{gathered}
\left\|P_{h} y-y_{h}\right\|_{L^{\infty}\left(L^{2}\right)}+\left\|\Pi_{h} \boldsymbol{p}-\boldsymbol{p}_{h}\right\|_{L^{\infty}\left(L^{2}\right)} \leq C h^{\frac{3}{2}}, \\
\left\|P_{h} z-z_{h}\right\|_{L^{\infty}\left(L^{2}\right)}+\left\|\Pi_{h} \boldsymbol{q}-\boldsymbol{q}_{h}\right\|_{L^{\infty}\left(L^{2}\right)} \leq C h^{\frac{3}{2}}, \\
\left\|\operatorname{div}\left(\Pi_{h} \boldsymbol{p}-\boldsymbol{p}_{h}\right)\right\|_{L^{2}\left(L^{2}\right)}+\left\|\operatorname{div}\left(\Pi_{h} \boldsymbol{q}-\boldsymbol{q}_{h}\right)\right\|_{L^{2}\left(L^{2}\right)} \leq C h^{\frac{3}{2}} .
\end{gathered}
$$

\section{Numerical Experiments}

In this section, we present a numerical example to validate our convergence and superconvergence results. The hyperbolic OCP was dealt numerically with codes developed based on AFEPack. The package is freely available and the details can be found in [33].

Let $\tau>0, N=T / \tau \in \mathbb{Z}$, and $t_{n}=n \tau, n=0,1, \cdots, N$. Set

$$
\phi^{n}=\phi\left(x, t_{n}\right), \phi_{t t}^{n}=\left(\phi^{n+1}-2 \phi^{n}+\phi^{n-1}\right) / \tau^{2} .
$$

Then, a fully discrete splitting positive definite mixed finite element solution $\left(\mathbf{p}_{h}^{n}, y_{h}^{n}, \mathbf{q}_{h}^{n}, z_{h}^{n}, u_{h}^{n}\right)$ of (1)-(6) satisfies the following system:

$$
\begin{aligned}
& \left(A^{-1} \boldsymbol{p}_{h, t \mathrm{t}}^{n}, \boldsymbol{v}_{h}\right)+\left(\operatorname{div} \boldsymbol{p}_{h}^{n}, \operatorname{div} \boldsymbol{v}_{h}\right)=\left(f^{n}, \operatorname{div} \boldsymbol{v}_{h}\right) \\
& +\left(u_{h}^{n}, \operatorname{div} \boldsymbol{v}_{h}\right), \forall \boldsymbol{v}_{h} \in V_{h}, n=1,2, \cdots, N . \\
& \left(\frac{\boldsymbol{p}_{h}^{1}-\boldsymbol{p}_{h}^{-1}}{2 \tau}, \boldsymbol{v}_{h}\right)=\left(\boldsymbol{p}_{t}(x, 0), \boldsymbol{v}_{h}\right), \forall \boldsymbol{v}_{h} \in \boldsymbol{V}_{h}, \\
& \boldsymbol{p}_{h}^{0}=\Pi_{h} \boldsymbol{p}(x, 0), \forall x \in \Omega, \\
& \left(y_{h, t t}^{n}, w_{h}\right)+\left(\operatorname{div} \boldsymbol{p}_{h}^{n}, w_{h}\right)=\left(f^{n}, w_{h}\right) \\
& +\left(u_{h}^{n}, w_{h}\right), \forall w_{h} \in W_{h}, n=1,2, \cdots, N, \\
& \left(\frac{y_{h}^{1}-y_{h}^{-1}}{2 \tau}, w_{h}\right)=\left(y_{1}(x), w_{h}\right), \forall w_{h} \in W_{h}, \\
& y_{h}^{0}=P_{h} y_{0}(x), \quad \forall x \in \Omega, \\
& \left(A^{-1} \boldsymbol{q}_{h, t t}^{n}, \boldsymbol{v}_{h}\right)-\left(\operatorname{div} \boldsymbol{q}_{h}^{n}, \operatorname{div} \boldsymbol{v}_{h}\right)=\left(\boldsymbol{p}_{h}^{n}-\boldsymbol{p}_{d}^{n}, \boldsymbol{v}_{h}\right) \\
& +\left(z_{h}^{n}, \operatorname{div} \boldsymbol{v}_{h}\right), \forall \boldsymbol{v}_{h} \in \boldsymbol{V}_{h}, n=N, N-1, \cdots, 1, \\
& \left(\frac{\boldsymbol{q}_{h}^{N-1}-\boldsymbol{q}_{h}^{N+1}}{2 \tau}, \boldsymbol{v}_{h}\right)=\left(\boldsymbol{q}_{t}(x, T), \boldsymbol{v}_{h}\right), \forall \boldsymbol{v}_{h} \in \boldsymbol{V}_{h} \text {, } \\
& \boldsymbol{q}_{h}^{N}=0, \forall x \in \Omega, \\
& \left(z_{h, t t}^{n}, w_{h}\right)=\left(y_{h}^{n}-y_{d}^{n}, w_{h}\right), \forall w_{h} \in W_{h}, n=N, N-1, \cdots, 1 \text {, } \\
& \left(\frac{z_{h}^{N-1}-z_{h}^{N+1}}{2 \tau}, w_{h}\right)=\left(z_{t}(x, T), w_{h}\right), \forall w_{h} \in W_{h}, \\
& z_{h}^{N}=0, \forall x \in \Omega,
\end{aligned}
$$

$u_{h}^{n}=\max \left\{0,-\left(z_{h}+\overline{\operatorname{div}} \boldsymbol{q}_{h}\right)\right\}+z_{h}^{n}+\operatorname{div} \boldsymbol{q}_{h}^{n}, \forall n=0,1, \cdots, N$.

Example 10. Let $\Omega=(0,1) \times(0,1), T=1, c(x)=1, A=E$, where $E$ denotes identity matrix. The data under testing are as follows:

$$
\begin{gathered}
y(x, t)=t^{2} \sin \left(2 \pi x_{1}\right) \sin \left(2 \pi x_{2}\right), \\
\boldsymbol{p}(x, t)=-\left(\begin{array}{c}
2 \pi t^{2} \cos \left(2 \pi x_{1}\right) \sin \left(2 \pi x_{2}\right) \\
2 \pi t^{2} \sin \left(2 \pi x_{1}\right) \cos \left(2 \pi x_{2}\right)
\end{array}\right), \\
\boldsymbol{q}(x, t)=\left(\begin{array}{c}
(1-t)^{2} \cos \left(2 \pi x_{1}\right) \sin \left(2 \pi x_{2}\right) \\
(1-t)^{2} \sin \left(2 \pi x_{1}\right) \cos \left(2 \pi x_{2}\right)
\end{array}\right), \\
z(x, t)=-\operatorname{div} \boldsymbol{q}(x, t)+2(1-t) \sin \left(2 \pi x_{1}\right) \sin \left(2 \pi x_{2}\right), \\
y_{d}(x, t)=y(x, t)-z_{t t}(x, t), \\
\boldsymbol{p}_{d}(x, t)=\boldsymbol{p}(x, t)-\boldsymbol{q}_{t t}(x, t)+\left(\begin{array}{c}
2 \pi(1-t) \cos \left(2 \pi x_{1}\right) \sin \left(2 \pi x_{2}\right) \\
2 \pi(1-t) \sin \left(2 \pi x_{1}\right) \cos \left(2 \pi x_{2}\right)
\end{array}\right), \\
u(x, t)=\max \{0,-z(x, t)+\overline{\operatorname{div}} \boldsymbol{q}(x, t)\}+z(x, t)+\operatorname{div} \boldsymbol{q}(x, t), \\
f(x, t)=y_{t t}(x, t)+\operatorname{div} \boldsymbol{p}(x, t)-u(x, t) .
\end{gathered}
$$

Let $e_{u}=u-u_{h}, e_{y}=y-y_{h}, e_{\mathbf{p}}=\boldsymbol{p}-\boldsymbol{p}_{h}, e_{z}=z-z_{h}, e_{\boldsymbol{q}}=\boldsymbol{q}$ - $\boldsymbol{q}_{h}$ and $E_{u}=P_{h} u-u_{h}, E_{y}=P_{h} y-y_{h}, E_{\mathbf{p}}=\Pi_{h} \boldsymbol{p}-\boldsymbol{p}_{h}, E_{z}=$ 
TABLE 2: Numerical results of convergence.

\begin{tabular}{ccccccccc}
\hline$h=\tau$ & $\left\|e_{p}\right\|_{\infty, 2}$ & Rate & $\left\|e_{q}\right\|_{\infty, 2}$ & Rate & $\left\|\operatorname{div} e_{p}\right\|_{2,2}$ & Rate & $\left\|\operatorname{div} e_{q}\right\|_{2,2}$ & Rate \\
\hline $1 / 10$ & $4.7647 e-2$ & - & $4.9557 e-2$ & - & $5.5145 e-2$ & - & $6.7192 e-2$ & - \\
$1 / 20$ & $2.4324 e-2$ & 00.97 & $2.5128 e-2$ & 0.98 & $2.8071 e-2$ & 0.97 & $3.3987 e-2$ & 0.98 \\
$1 / 40$ & $1.2153 e-2$ & 1.00 & $1.28077 e-2$ & 0.97 & $1.4045 e-2$ & 1.00 & $1.6943 e-2$ & 1.00 \\
$1 / 80$ & $6.0748 e-3$ & 1.00 & $6.4102 e-3$ & 1.00 & $7.0231 e-3$ & 1.00 & $8.4158 e-3$ & 1.01 \\
\hline
\end{tabular}

TABLE 3: Numerical results of superconvergence.

\begin{tabular}{lccccccc}
\hline$h$ & $\tau$ & $\left\|E_{u}\right\|_{2,2}$ & Rate & $\left\|E_{y}\right\|_{\infty, 2}$ & Rate & $\left\|E_{z}\right\|_{\infty, 2}$ & Rate \\
\hline $1 / 10$ & $1 / 10$ & $4.8542 e-2$ & - & $3.4063 e-2$ & - & $4.0284 e-2$ & - \\
$1 / 20$ & $1 / 30$ & $1.7282 e-2$ & 1.49 & $1.2154 e-2$ & 1.49 & $1.4404 e-2$ & 1.48 \\
$1 / 40$ & $1 / 90$ & $6.0473 e-3$ & 1.51 & $4.3104 e-3$ & 1.50 & $5.0486 e-3$ & 1.51 \\
$1 / 80$ & $1 / 270$ & $2.1158 e-3$ & 1.52 & $1.4835 e-3$ & 1.54 & $1.7635 e-3$ & 1.52 \\
\hline
\end{tabular}

TABLE 4: Numerical results of superconvergence.

\begin{tabular}{cccccccccc}
\hline$h$ & $\tau$ & $\left\|E_{p}\right\|_{\infty, 2}$ & Rate & $\left\|E_{q}\right\|_{\infty, 2}$ & Rate & $\left\|\operatorname{div} E_{p}\right\|_{2,2}$ & Rate & $\left\|\operatorname{div} E_{q}\right\|_{2,2}$ & Rate \\
\hline $1 / 10$ & $\frac{1}{10}$ & $4.6684 e-2$ & - & $4.7694 e-2$ & - & $5.4134 e-2$ & - & $6.5048 e-2$ & - \\
$1 / 20$ & $1 / 30$ & $1.6902 e-2$ & 1.47 & $1.7120 e-2$ & 1.48 & $1.9402 e-2$ & 1.48 & $2.3406 e-2$ & 1.47 \\
$1 / 40$ & $1 / 90$ & $5.9615 e-3$ & 1.50 & $6.0452 e-3$ & 1.50 & $6.8342 e-3$ & 1.51 & $8.1906 e-3$ & 1.51 \\
$1 / 80$ & $1 / 270$ & $2.0512 e-3$ & 1.54 & $2.1124 e-3$ & 1.52 & $2.3448 e-3$ & 1.54 & $2.8302 e-3$ & 1.53 \\
\hline
\end{tabular}

$P_{h} z-z_{h}, E_{\boldsymbol{q}}=\Pi_{h} \boldsymbol{q}-\boldsymbol{q}_{h}$. We set $\|\cdot\|_{L^{\infty}\left(L^{2}\right)}=\|\cdot\|_{\infty, 2}$ and $\|\cdot\|_{L^{2}\left(L^{2}\right)}=\|\cdot\|_{2,2}$. The numerical results of convergence and superconvergence based on a sequence of uniformly refined meshes are reported in Tables 1-4, respectively. They are consistent with our theoretical results.

\section{Data Availability}

The data used to support the findings of this study are included within the article.

\section{Conflicts of Interest}

The authors declare that there are no conflicts of interest.

\section{Acknowledgments}

The first author is supported by the Scientific Research Project of Hunan Provincial Department of Education (20C0854) and the Scientific Research Program in Hunan University of Science and Engineering (20XKY059). The second author is supported by the National Natural Science Foundation of China (11401201), the Natural Science Foundation of Hunan Province (2020JJ4323), the Scientific Research Project of Hunan Provincial Department of Education (20A211), and the Construct Program of Applied Characteristic Discipline in Hunan University of Science and Engineering.

\section{References}

[1] Y. Chen and Y. Dai, "Superconvergence for optimal control problems governed by semi-linear elliptic equations," Journal of Scientific Computing, vol. 39, no. 2, pp. 206-221, 2009.

[2] R. Li, W. Liu, H. Ma, and T. Tang, "Adaptive finite element approximation for distributed elliptic optimal control problems," SIAM Journal on Control and Optimization, vol. 41, no. 5, pp. 1321-1349, 2002.

[3] C. Meyer and A. Rösch, "Superconvergence properties of optimal control problems," SIAM Journal on Control and Optimization, vol. 43, no. 3, pp. 970-985, 2004.

[4] C. Meyer and A. Rösch, " $L^{\infty}$-error estimates for approximated optimal control problems," SIAM Journal on Control and Optimization, vol. 44, no. 5, pp. 1636-1649, 2005.

[5] D. Yang, Y. Chang, and W. Liu, "A priori error estimates and superconvergence analysis for an optimal control problems of bilinear type," Journal of Computational Mathematics, vol. 4, pp. 471-487, 2008.

[6] N. Yan, "Superconvergence analysis and a posteriori error estimation of a finite element method for an optimal control problem governed by integral equations," Applications of Mathematics, vol. 54, no. 3, pp. 267-283, 2009.

[7] D. Meidner and B. Vexler, "A priori error estimates for spacetime finite element discretization of parabolic optimal control problems part II: problems with control constraints," SIAM Journal on Control and Optimization, vol. 47, no. 3, pp. 1301-1329, 2008. 
[8] R. S. McKnight and W. E. Bosarge Jr., "The Ritz-Galerkin procedure for parabolic control problems," SIAM Journal on Control and Optimization, vol. 11, no. 3, pp. 510-524, 1973.

[9] L. Hou and J. Turner, "Analysis and finite element approximation of an optimal control problem in electrochemistry with current density controls," Numerische Mathematik, vol. 71, no. 3, pp. 289-315, 1995.

[10] G. Knowles, "Finite element approximation of parabolic time optimal control problems," SIAM Journal on Control and Optimization, vol. 20, no. 3, pp. 414-427, 1982.

[11] Y. Tang and Y. Chen, "Variational discretization for parabolic optimal control problems with control constraints," Journal of Systems Science and Complexity, vol. 25, no. 5, pp. 880-895, 2012.

[12] Y. Tang and Y. Chen, "Superconvergence of finite element methods for optimal control problems governed by parabolic equations with time-dependent coefficients," East Asian Journal on Applied Mathematics, vol. 3, no. 3, pp. 209-227, 2013.

[13] Y. Tang and Y. Hua, "Superconvergence analysis for parabolic optimal control problems," Calcolo, vol. 51, no. 3, pp. 381-392, 2014.

[14] B. Desmorat, M. Spagnuolo, and E. Turco, "Stiffness optimization in nonlinear pantographic structures," Mathematics and Mechanics of Solids, vol. 25, no. 12, pp. 2252-2262, 2020.

[15] I. Giorgio, "Lattice shells composed of two families of curved Kirchhoff rods: an archetypal example, topology optimization of a cycloidal metamaterial," Continuum Mechanics and Thermodynamics, vol. 33, no. 4, pp. 1063-1082, 2021.

[16] Y. Chen, Y. Huang, W. Liu, and N. Yan, "Error estimates and superconvergence of mixed finite element methods for convex optimal control problems," Journal of Scientific Computing, vol. 42, no. 3, pp. 382-403, 2009.

[17] Y. Chen and Z. Lu, High Efficient and Accuracy Numerical Methods for Optimal Control Problems, Science Press, Beijing, 2015.

[18] Y. Chen, Z. Lu, and Y. Huang, "Superconvergence of triangular Raviart-Thomas mixed finite element methods for a bilinear constrained optimal control problem," Computers \& Mathematcs with Applications, vol. 66, no. 8, pp. 1498-1513, 2013.

[19] Z. Lu and S. Zhang, " $L^{\infty}$-error estimates of rectangular mixed finite element methods for bilinear optimal control problem," Applied Mathematics and Computation, vol. 300, pp. 79-94, 2017.

[20] X. Xing and Y. Chen, "Superconvergence of mixed methods for optimal control problems governed by parabolic equations," Advances in Applied Mathematics and Mechanics, vol. 3, no. 4, pp. 401-419, 2011.

[21] D. Yang, "A splitting positive definite mixed element method for miscible displacement of compressible flow in porous media," Numerical Methods for Partial Differential Equations: An International Journal, vol. 17, no. 3, pp. 229-249, 2001.

[22] Y. Liu, H. Li, J. Wang, and S. He, "Splitting positive definite mixed element methods for pseudo-hyperbolic equations," Numerical Methods for Partial Differential Equations: An International Journal, vol. 28, no. 2, pp. 670-688, 2012.

[23] J. Zhang and D. Yang, "A splitting positive definite mixed element method for second-order hyperbolic equations," Numerical Methods for Partial Differential Equations: An International Journal, vol. 25, no. 3, pp. 622-636, 2009.

[24] H. Guo, H. Fu, and J. Zhang, "A splitting positive definite mixed finite element method for elliptic optimal control prob- lem," Applied Mathematics and Computation, vol. 219, no. 24, pp. 11178-11190, 2013.

[25] Y. Tang and Y. Hua, "Superconvergence of splitting positive definite mixed finite element for parabolic optimal control problems," Applicable Analysis, vol. 97, no. 16, pp. 27782793, 2018.

[26] C. Xu, "A priori error estimates and superconvergence of splitting positive definite mixed finite element methods for pseudohyperbolic integro-differential optimal control problems," Numerical Analysis and Applications, vol. 13, no. 1, pp. 1733, 2020.

[27] Z. Lu, L. Li, Y. Feng, L. Cao, and W. Zhang, "Error estimates of finite volume element method for nonlinear hyperbolic optimal control problems," Italian Journal of Pure and Applied Mathematics, vol. 41, pp. 70-84, 2019.

[28] J. Lions, Optimal Control of Systems Governed by Partial Differential Equations, Springer-Verlag, Berlin, 1971.

[29] J. Douglas and J. Roberts, "Global estimates for mixed methods for second order elliptic equations," Mathematics of Computation, vol. 44, no. 169, pp. 39-52, 1985.

[30] P. Raviart and J. Thomas, "A mixed finite element method for 2nd order elliptic problems," Aspecs of the Finite Element Method, Lecture Notes in Math., vol. 606, pp. 292-315, 1977.

[31] F. Brezzi and M. Fortin, Mixed and Hybrid Finite Element Methods, Springer-Verlag, New York, 1991.

[32] R. Ewing, M. Liu, and J. Wang, "Superconvergence of mixed finite element approximations over quadrilaterals," SIAM Journal on Numerical Analysis, vol. 36, no. 3, pp. 772-787, 1999.

[33] R. Li and W. Liu, "Software introduction: AFEPack," https:// blog.csdn.net/HateCode/article/details/1413290. 